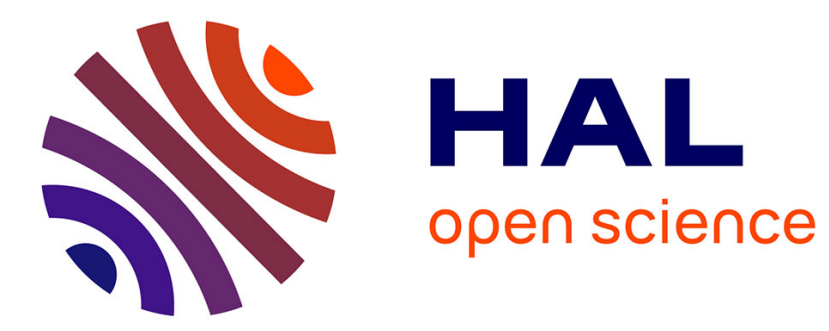

\title{
Curves Matching Using Geodesic Paths
}

Isaac Cohen, Isabelle Herlin

\section{To cite this version:}

Isaac Cohen, Isabelle Herlin. Curves Matching Using Geodesic Paths. Proceedings of the conference on Computer Vision and Pattern Recognition, IEEE, Jun 1998, Santa-Barbara, United States. pp.741746. inria-00532724

\section{HAL Id: inria-00532724 https://inria.hal.science/inria-00532724}

Submitted on 20 Apr 2016

HAL is a multi-disciplinary open access archive for the deposit and dissemination of scientific research documents, whether they are published or not. The documents may come from teaching and research institutions in France or abroad, or from public or private research centers.
L'archive ouverte pluridisciplinaire HAL, est destinée au dépôt et à la diffusion de documents scientifiques de niveau recherche, publiés ou non, émanant des établissements d'enseignement et de recherche français ou étrangers, des laboratoires publics ou privés. 


\section{Curves Matching Using Geodesic Paths}

\author{
Isaac COHEN \\ icohen@iris.usc.edu \\ Institute for Robotics and Intelligent Systems \\ University of Southern California \\ PHE 204 MC-0273 \\ Los Angeles, CA 90089-0273
}

\author{
Isabelle HERLIN \\ Isabelle.Herlin@inria.fr \\ INRIA \\ Projet AIR \\ B.P. 78153 Le Chesnay CEDEX \\ France
}

\begin{abstract}
We present a method for matching curves which accommodates large and small deformation. The method preserves geometric similarities in the case of small deformation, and loosens these geometric constraints when large deformations occur. The approach is based on the computation of a set of geodesic paths connecting the curves. These two curves are defined as a source area and a destination area which can have an arbitrary number of connected components and different topologies. The applicative framework of the presented method is the study of the crustal deformation from a set of iso-elevation curves. An experiment with real curves demonstrates that the approach can be successfully applied to characterize deformation of Digital Elevation Models.

Keywords: Curves matching, motion analysis, geodesic distance computation, Eulerian formulation, geometric properties.
\end{abstract}

\section{Introduction}

The use of remote sensing data to study natural phenomena is increasing and represent a new trend in computer vision. Indeed, most of natural phenomena can be monitored through a dedicated remote sensing device and usually involves processing of large image sequences. In this paper we are particularly interested in studying and measuring earth crustal deformation from a Synthetic Aperture Radar (SAR) image sequence. SAR data allow to create a Digital Elevation Model (DEM) by unwarping the interferometric image generated from a pair of acquisitions. Moreover, using more than two SAR images and a DEM of the studied site, we can also derive differential interferometric images which represent the observed surface deformation [6]. However, such images are corrupted with noise and are difficult to use and therefore a more reliable approach consists in characterizing crustal deformation from the DEM of the monitored site.

In this paper we propose a new approach for studying the crustal deformation based on a characterization of the deformation from the DEM available before and after an earthquake. The proposed approach is based on matching features extracted from the two DEM. We use a pointwise tracking approach to match these structures. More precisely, the deformation between the two sets of feature points is characterized through a set of paths connecting the feature points. This approach accommodates for large and small deformations in order to preserve geometric similarities in the case of small deformation and loosen the geometric properties when large deformation occurs. Such an approach allows to merge, in a single scheme, the methods based on invariant geometrical properties which apply in the case of small deformation and those dealing with large deformation.

\subsection{Previous Works}

Studying the evolution of structures represented through their boundaries requires to match each point belonging to these curves. The deformation of the structure is then infered from this point to point matching. Several issues have to be addressed such as the definition of feature points which will be used as landmarks and the definition of the matching function that defines the conjugate pairs of points.

Points of high curvature are commonly used as feature points. Indeed, in the case of rigid, affine or small elastic deformation $[4,9]$ the high curvature points or semi-differential invariants [11] can be considered as invariant description of the curves. These invariants are matched according to the nature of the deformation through the use of an appropriate similarity measure. The output of such approaches are a set of conjugate pairs of points belonging to the matched curves. These approaches fail in the case of non parametric deformations or deformations of large amplitude. Moreover, in some situations the description of the structures to be matched cannot be obtained easily. Indeed, these methods rely on a good representation of the structures and have poor performances when a curve contains corners and cusps, or when a curve cannot be parameterized by a single smooth function.

A second issue in curves matching is the topology of the studied curves. When these curves have different topology, most of the methods fail since they assume implicitly that the surface generated by the curves does not have singular points or saddle points $[1,11]$. Such singularities are observed when matching curves which undergo a large deformation.

\subsection{Matching Curves of Arbitrary Topology}

In this paper we propose an extension of the approach previously proposed by Cohen et al [5] and based on the computation of a set of paths connecting the two structures. The key 
point of the method is the computation of the set of geodesic paths The curves are defined as a source area $S$ and a destination area $D$. Matching the source and destination areas is done through the computation of paths connecting these regions. These paths are defined on a graph surface and minimize a cost function which measures the similarity between the two areas. This approach is particularly attractive in the absence of a reliable geometrical information and allows to match curves which undergo a large deformation or curves with a complex and variable topology.

Our geodesic approach allows to take into account geometrical properties of the matched curves when a small deformation is detected and use geodesic distance maps simultaneously for large deformation. This approach gives consideration to large and small deformation in order to preserve geometric similarities in the case of small deformations and relax these geometric constraints when large deformations occur. Such an approach allows to merge, in a single framework, the methods based on invariant geometrical properties which apply in the case of small deformations and those dealing with large deformations.

The structures to match are defined as a source area $S$ and a destination area $D$. A geodesic distance map is derived for each of these areas as described in section 2. These maps are used to derive a similarity measure allowing to match the source and destination area. The geodesic maps naturally depend on the graph surface on which they lie. A description of different possible graph surfaces is given in section 3 . The definition of the graph surface can be more elaborate in order to take into account the geometric properties of the structures to match. Section 4 describes the selected approach. The geodesic paths matching these structures are computed using a local minimization of the similarity measure. This step is described in section 5. In section 6 an experimental result obtained on iso-elevation curves illustrates that the approach can be successfully applied to characterize crustal deformation.

\section{Geodesic Distance Computation}

Given a graph surface $Z=(x, y, z(x, y))$, and a set of points lying on the graph surface $Z$ and represented through a level set equation $\varphi^{-1}(0)$ the geodesic distance map from this set of points can be derived by solving the following propagation equation [7]:

$$
\varphi_{t}=\sqrt{a \varphi_{x}^{2}+b \varphi_{y}^{2}-c \varphi_{x} \varphi_{y}}
$$

where $a=\frac{1+q^{2}}{1+p^{2}+q^{2}}, b=\frac{1+p^{2}}{1+p^{2}+q^{2}}, c=\frac{2 p q}{1+p^{2}+q^{2}}$ and $p=\frac{\partial z}{\partial x}, q=\frac{\partial z}{\partial y}$ are the partial derivatives of $z(x, y)$. This scheme allows us to compute easily the geodesic distance map through a control of the speed function of the propagation according to the graph surface geometric properties reflected by the parameters $a, b$ and $c$. Indeed, the speed function controlling the propagation equation (1) combines the first fundamental forms of the graph surface $Z$ and of the surface $\varphi$.
The initial estimate $\varphi_{0}$ corresponds to the level set representation of the set of points lying on the surface $Z$. This implicit representation has to satisfy the following requirements: the initial estimate has to be smooth, negative in the interior of the domain defined by the boundary $\varphi_{0}^{-1}(0)$ and positive otherwise. Computing such an initial estimate can be done easily. Indeed, one can use a signed distance computation [3] in order to satisfy the previous requirements. The following function, defined by:

$$
\varphi_{0}(x, y)= \begin{cases}-d(x, y) & \text { if }(x, y) \in \text { interior of } \varphi_{0}^{-1}(0) \\ 0 & \text { if }(x, y) \in \varphi_{0}^{-1}(0) \\ d(x, y) & \text { if }(x, y) \in \text { exterior of } \varphi_{0}^{-1}(0)\end{cases}
$$

can be used as an initial estimate for solving the propagation equation (1).

Given a graph surface $Z$ and the initial estimate $\varphi_{0}$ (eq. (2)) on this surface, equation (1) characterizes the distance map on the graph surface $Z$ of the area which boundary is defined by $\varphi_{0}^{-1}(0)$.

A solution of equation (1) can be obtained through a numerical schemes used for Hamilton Jacobi equations. We have used an explicit temporal scheme, while the spatial gradients are derived through a finite difference approximation and the minmod method which selects the smallest slope between the forward and backward derivatives $[5,7,10]$.

Describing the set of points lying on the surface $Z$ using a level set equation allows us to model curves with different topologies or structures with isolated points or cusps. Furthermore, such a formulation insures a good numerical stability and accuracy.

Recently, Cohen et al [5] introduced a new method for pointwise tracking of structures by matching their contours. The deformation between two temporal occurrences is obtained through a set of trajectories provided by the matching process. The method is based on the computation of a set of paths connecting the two curves to be matched. Each path minimizes a cost function which measures the local similarity between the starting and ending points of the path. An important issue of the approach proposed in [5] is the definition of the graph surface on which are computed the geodesic distance maps. In section 3 we describe different graph surfaces that can be used and extend, in section 4, the work presented in [5] by defining a new graph surface taking into account local properties of the curves in the matching method.

\section{Definition Of The Graph Surface}

Matching two sets of points or two structures using a geodesic distance map approach, requires that the geodesic distance maps associated to each set of points, the source $S$ and the destination $D$, have to be computed using the same graph surface $Z$. Henceforth, the the source $S$ and the destination $D$ points must lie on the same graph surface $Z$.

We will now give two definitions of such a graph surface and illustrate the different results on synthetical data. Let first denote by $S=\left(x, y, \varphi_{0}(x, y)\right)$ the source area, $D=$ 


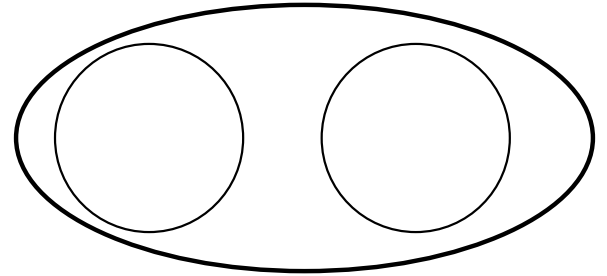

Figure 1: A plot of the structures that have to be matched. The first structure composed of the circles is matched to an ellipse.

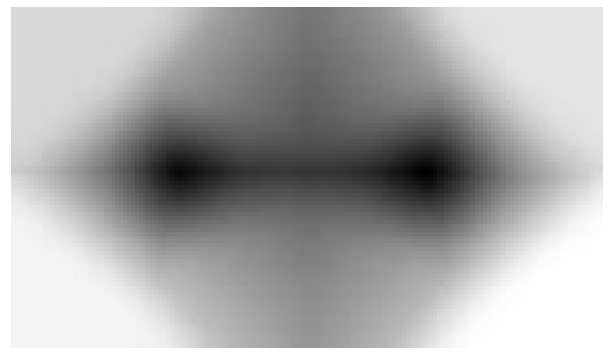

Figure 2: The graph surface $Z$ associated to the circles and the ellipse derived from eq. (5).

$\left(x, y, \psi_{0}(x, y)\right)$ the destination area and $\mathcal{D}_{S}$ and $\mathcal{D}_{D}$ denote the geodesic distance maps obtained by solving equation (1). These maps are defined by the equations:

$$
\mathcal{D}_{S}=\{(x, y, \varphi(x, y))\}
$$

and,

$$
\mathcal{D}_{D}=\{(x, y, \psi(x, y))\}
$$

where $\varphi$ and $\psi$ are respectively the solutions of equation (1) with initial estimate $\varphi_{0}$ and $\psi_{0}$. The $\varphi$ and $\psi$ functions represent the distance maps on the graph surface $Z$ of the source and destination areas.

Defining a graph surface $Z$ such that the two initial curves $\varphi_{0}$ and $\psi_{0}$ lye on the same surface can be done easily using the minimal value of the distance or the product of the distance maps. In both cases, the initials curves are zero level set curves of the new graph surface. The following equation:

$$
Z=(x, y, z(x, y))=\left(x, y, \min \left(\left|\varphi_{0}\right|,\left|\psi_{0}\right|\right)\right) .
$$

defines a graph surface on which the source and destination areas are zero level sets. This surface preserves the distance map and represents, at each point, the distance to the nearest structure.

Another graph surface can be obtained using the following definition:

$$
Z=(x, y, z(x, y))=\left(x, y,\left|\varphi_{0} \| \psi_{0}\right|\right)
$$

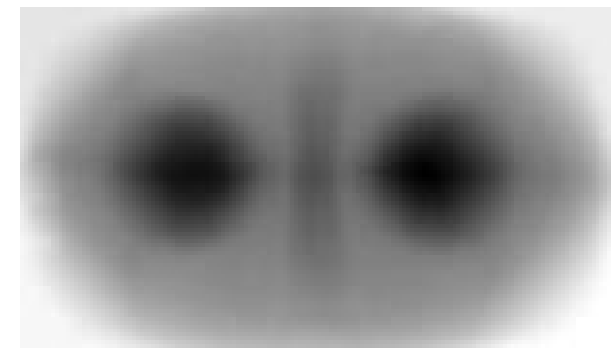

Figure 3: The graph surface $Z$ associated to the circles and the ellipse derived from eq. (6).

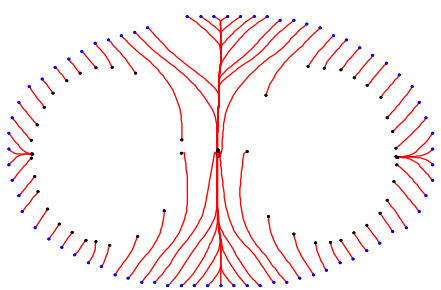

Figure 4: Paths connecting the two structures derived from the graph surface defined by equation (5).

In this second definition, the distance maps are altered and introduce some geometric changes in the graph surface which are not related to the geometric properties of the structures to be matched. These artefacts are displayed in figure 3 are mainly due to the relative distance between the structures to be matched and do not reflect any shape properties of the curves.

These two definitions are illustrated on a synthetic example displayed in figure 1. In this example we match an ellipse with two circles. The graph surface $Z$ defined by the minimal distance or the product of the distance are shown respectively in figures 2 and 3. Naturally, the geodesic paths matching the two structures vary according to the graph surface on which they are computed. We display in figure 4 and 5 the matching obtained by considering, respectively the graph surface defined by equations (5) and (6). We can observe that using the graph surface defined by (6) changes considerably the paths due to the shape of the graph surface displayed in figure 3. The properties of the graph surface defined by (6) are not suitable for a matching method since the graph surface is more dependent on the euclidean distance map of each structure rather than the relative distance between these structures. We will therefore, use in the following the graph surface defined by equation (5).

\section{Modeling Geometric Properties}

In the previous definitions of the graph surface, the geometric properties of the curves to be matched are not taken into 


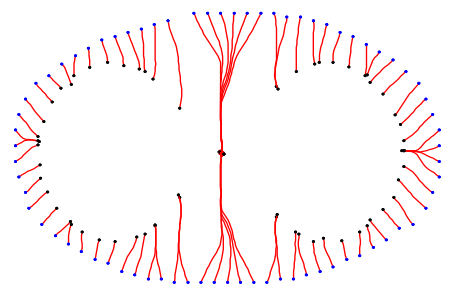

Figure 5: Paths connecting the two structures derived from the graph surface defined by equation (6).

account. These definitions are based on the distance maps of each structure regardless of its local properties.

In this section we define a new graph surface which adapt itself to large and small deformations in order to preserve geometric similarities in the case of small deformations and loosens these geometric constraints when large deformations occur. Such an approach allows to merge, in a single framework, the methods based on invariant geometrical properties which apply in the case of small deformation and those dealing with large deformation.

This more elaborate definition of the graph surface also takes into account the geometrical properties of the curves. For example, we can use the curvature information in a small neighborhood of the source and destination areas. Within this neighborhood curvature information is relevant since only small deformation occurs and their curvature measure may be used to strengthen the matching of similar points.

We characterize the local properties of the structures through the principal curvatures $k_{S}$ and $k_{D}$ of the surface $S$ and $D$ respectively. In the case of small deformation we want the graph surface $Z$ to reflect the similarity between points having a similar principal curvatures. This property will be down-weight as the distance increases. For that purpose, we use the following graph surface

$$
\begin{aligned}
Z=(x, y, \min & \left(\left(1-\frac{\left(k_{S}-k_{D}\right)^{2}}{1+\varphi_{0}^{2}\left(k_{S}-k_{D}\right)^{2} / W^{2}}\right) \varphi_{0},\right. \\
& \left.\left.\left(1-\frac{\left(k_{S}-k_{D}\right)^{2}}{1+\psi_{0}^{2}\left(k_{S}-k_{D}\right)^{2} / W^{2}}\right) \psi_{0}\right)\right)
\end{aligned}
$$

where $W$ defines the size of the neighborhood on which the geometric properties have to be taken into account. The principal curvature is defined by the following equation:

$$
k=\frac{H+K}{2}
$$

where $H$ and $K$ are respectively the mean curvature and gaussian curvature of the surface defined by the following equations:

$$
H=\frac{\left(1+\phi_{x x}\right) \phi_{y}^{2}-2 \phi_{x} \phi_{y} \phi_{x y}+\left(1+\phi_{y y}\right) \phi_{x}^{2}}{\left(1+\phi_{x}^{2}+\phi_{y}^{2}\right)^{3 / 2}}
$$

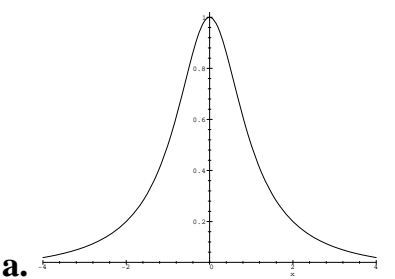

b.

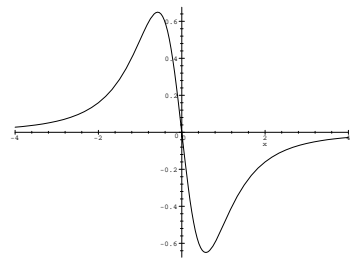

Figure 6: Robust error norm $\rho$ and its derivative.

and

$$
K=\frac{\phi_{x x} \phi_{y}^{2}-2 \phi_{x} \phi_{y} \phi_{x y}+\phi_{y y} \phi_{x}^{2}}{\left(\phi_{x}^{2}+\phi_{y}^{2}\right)^{3 / 2}}
$$

The definition of the graph surface given by equation (7) rely on the robust error norm $\rho$ defined by:

$$
\rho(x, d)=1-\frac{x^{2}}{1+d^{2} x^{2} / \sigma}
$$

where $\sigma$ is a scale parameter. Indeed, the graph surface (7) is given by the following equation:

$$
Z=\left(x, y, \min \left(\varphi_{0} \rho\left(k_{S}-k_{D}, \varphi_{0}\right), \psi_{0} \rho\left(k_{S}-k_{D}, \psi_{0}\right)\right)\right)
$$

The use of such a function (displayed in figure 6.a with $d=1$ ) to measure the similarity of the geometric properties allows reject or down-weight large dissimilarities. A set of robust error functions having the same behavior can be found in [8]. The derivative of this function (figure 6.b) characterizes the influence of residuals [2]. As the dissimilarities grow, the function $\rho$ decreases and the function $\rho$ approaches zero. In equation (7), we also make use of the Euclidean distance maps $\varphi_{0}$ and $\psi_{0}$ in order to define the range over which the curvature similarity is considered. Indeed, when the distance is large, the function $\rho$ approaches one and therefore only the distance to the nearest point is taken into account in equation (7). The graph function defined by equation (7) then converges to the one given by equation (5). The definition of the graph surface given by equation (7) allows to deal with large and small deformations in order to preserve geometric similarities in the case of small deformation and down-weight these geometric properties when large deformation occurs.

\section{Defining The Optimal Paths}

The matching method is based on the computation of a set of paths connecting the two curves to be matched. Each path minimize a cost function which measures the local similarity between the starting and ending points of the path. Consequently, the definition of a similarity measure amounts to locally define a cost function which relates the starting and ending points of a matching path. The cost along a path $p$ starting at a point $X_{S}$ and ending at $X_{D}$ is defined by:

$$
C\left(p_{X_{S}}\right)=\int_{X_{S}}^{X_{D}} f(x, y) d s
$$


where $f$ is a cost function, measuring the similarity between the points $X_{S}$ and $X_{D}$ which is the first point of the area $D$ attained by the path $p_{X_{S}}$.

The cost function $f$ has to characterize the similarity between the two curves. Furthermore, the matching between the source and destination area has to be symmetric in the sense that the source and destination areas may be inverted without changing the matching paths. This means that if $\tilde{p}_{X_{S}}^{X_{D}}$ denotes the optimal path connecting the points $X_{S}$ and $X_{D}$, we have the property: $\tilde{p}_{X_{S}}^{X_{D}}=\tilde{p}_{X_{D}}^{X_{S}}$. Fulfilling such a property restricts the choice of the cost function $f$, since it must define a similarity measure which depends only on the source and destination areas independently of the order in which they are considered.

We use the following cost function:

$$
f(x, y)=\varphi(x, y)+\psi(x, y)
$$

where $\varphi$ and $\psi$ are the bivariate functions defining the distance maps $\mathcal{D}_{S}$ and $\mathcal{D}_{D}$. This function allows to compute at each point of the plane the minimal distance on the surface $Z$ defined by equation (5) to the nearest region $S$ or $D$.

Defining the matching paths connecting the two areas amounts to characterize for each point $X_{S}$ on the first curve an optimal path $p^{\text {opt }}$ connecting this point to an unknown point $X_{D}$ on the second curve. This optimal path is defined by:

$$
C\left(p_{X_{S}}^{o p t}\right)=\min _{p_{X_{S}}} C\left(p_{X_{S}}\right) .
$$

Such an approach is not straightforward, since we have to compute all paths connecting the point $X_{S}$ to the destination area $D$ and then select the one with minimal cost. It is numerically expensive and one has to store all the paths before selecting the one with minimal cost value. Instead, we use a property of the graph surfaces $\mathcal{D}_{S}$ and $\mathcal{D}_{D}$ which relates the equal distance contour to minimal paths: these minimal paths are orthogonal to equal distance contours. Given a starting point $X_{S}$, this property defines a robust method for characterizing the path of minimal cost connecting this point to a point belonging to the destination area: such a path is characterized through its tangent vector. Since the equal distance contours are level sets of the map $\mathcal{D}_{S}+\mathcal{D}_{D}$, the tangent vector is defined by the gradient of the cost function:

$$
\nabla f=\nabla \varphi+\nabla \psi
$$

This approach gives a reliable path construction scheme: given a point $X_{S}$ on the source area, move this point in the opposite direction of the gradient of $f$ until reaching a point on the destination area. The result is the minimal cost path connecting the two areas and starting at $X_{S}$. This path is defined by the parameterized curve $p(s)$ such that: $p(0)=X_{S}$, $p(1)=X_{D}$ and

$$
\frac{\partial p}{\partial s}=-\nabla\left(\mathcal{D}_{S}+\mathcal{D}_{D}\right)
$$

where $X_{S} \in \varphi_{0}^{-1}(0)$ is given and $X_{D} \in \psi_{0}^{-1}(0)$ is unknown. This scheme is illustrated in figures 4,5 and 9 .

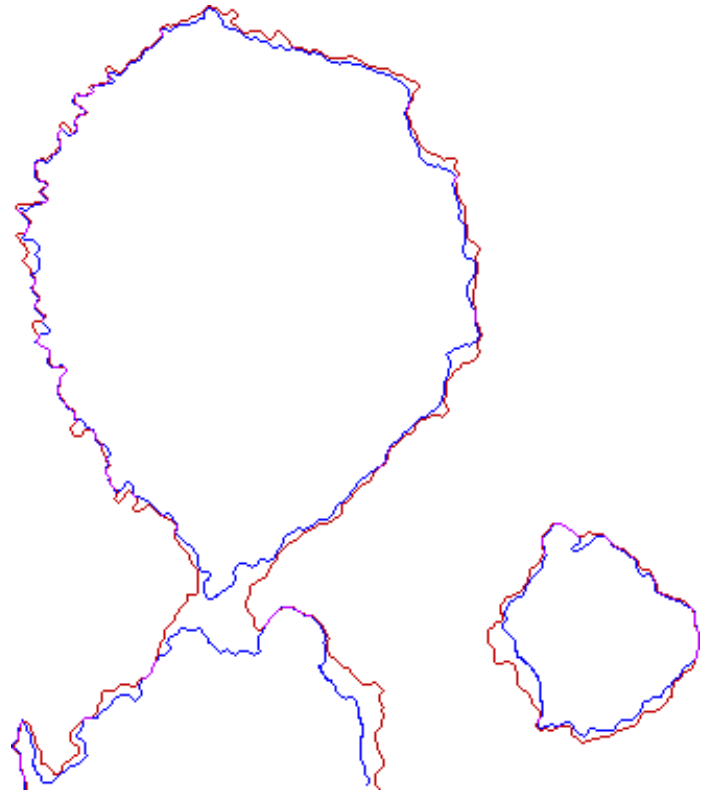

Figure 7: A plot of the iso-elevation curves taken before and after the eruption.

\section{Experimental Results}

The applicative framework of the presented method is the study of the crustal deformation from SAR image sequences. These images allow to derive DEM of the site and are therefore very useful for studying the crustal deformation since we can use two DEM, before and after the event, which reflect the deformation. This deformation is then studied by matching iso-elevation curves extracted from the two DEM.

Figure 7 illustrates the iso-elevation structures considered in studying the crustal deformation after Vatnajokull, Europe's largest glacier, eruption. The eruption melts the ice above the fissure, and a depression is created in the glacier surface, up to 2-300 $\mathrm{m}$ deep and $2 \mathrm{~km}$ wide.

The structures shown in 7 represent the source and destination areas to be matched. This example is very interesting since, we have simultaneously: large deformations at specific locations, small deformations, structures which are not connected and having different topologies. Moreover, the geometric properties are significant since they correspond to terrain features that should be preserved in the case of small deformation. The matching paths are displayed on figure 9. These paths do not intersect one with another. Furthermore, moving the blue particles along these paths gives us a way to synthetically create the temporal occurrences in between the source and destination structures.

\section{Conclusion}

We presented a curves matching method allowing to take into account geometrical properties of the matched curves when a small deformation is detected and to use geodesic distance maps for large deformation. This approach, based on 


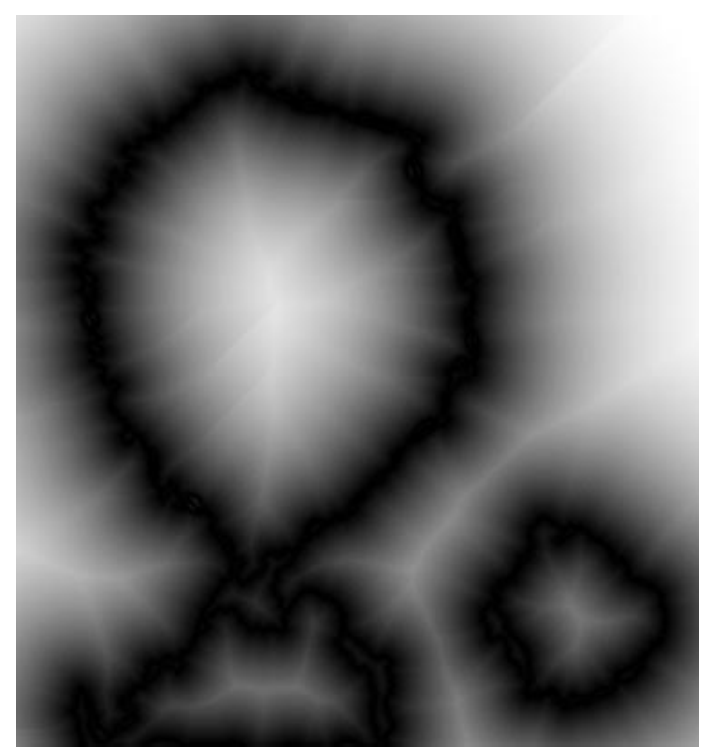

Figure 8: Cost function derived from the two iso-elevation curves to match

a robust error norm, accommodates large and small deformations in order to preserve geometric similarities in the case of small deformation and loosens these geometric constraints when large deformations occur. Such an approach allows to merge, in a single approach, the methods based on invariant geometrical properties which apply in the case of small deformation and those dealing with large deformation. Furthermore, the presented approach performs well in situations where we simultaneously have: large deformation at specific locations, small deformation, structures which are not connected and having different topologies.

\section{References}

[1] J.P. Berroir, I. Herlin, and I. Cohen. A numerical model for large deformation on meteorological images. In International Conference on Analysis and Optimisation of Systems, Images, Wavelets and PDE's, Paris, Ministère de la Recherche, Juin 1996.

[2] M. J. Black. Recursive non-linear estimation of discontinuous flow fields. In Third European Conference on Computer Vision, pages 138-145, Sweden, May 1994. Springer-Verlag.

[3] G. Borgefors. Distance transformations in arbitrary dimensions. Computer Vision, Graphics, and Image Processing, 27:321-345, 1984.

[4] I. Cohen, N. Ayache, and P. Sulger. Tracking points on deformable objects using curvature information. In Proceedings of the Second European Conference on Computer Vision 1992, pages 458-466, Santa Margherita Ligure, Italy, May 1992.

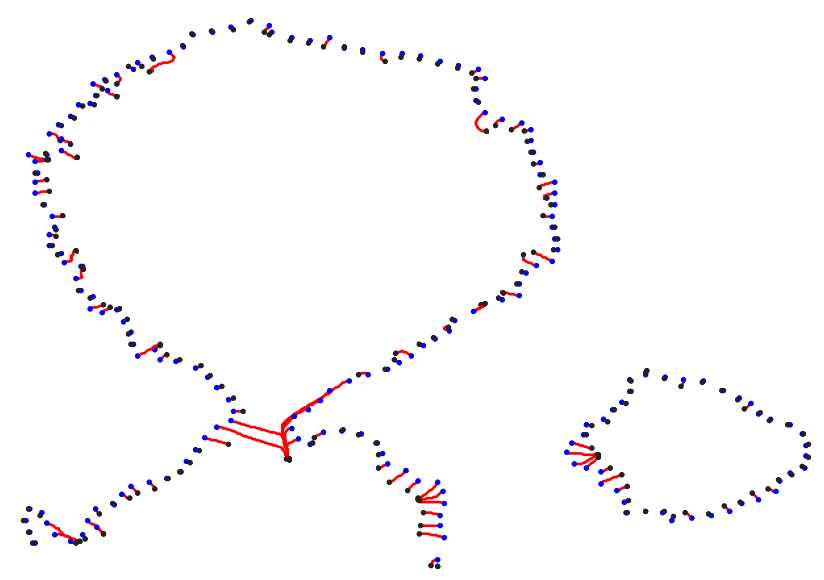

Figure 9: Paths matching the iso-elevation curves of the Vatnajokull.

[5] I. Cohen and I. Herlin. Tracking meteorological structures through curves matching using geodesic paths. In IEEE Proceedings of the International Conference on Computer Vision, Bombay, India, January 1998.

[6] E. Huot, I. Cohen, and I. Herlin. An unwrapping method for interferometric sar images. In ICASSP'97, Munich, Germany, 1997.

[7] R. Kimmel, A. Amir, and A.F. Bruckstein. Finding shortest paths on surfaces using levelset propagation. IEEE Transactions on Pattern Analysis and Machine Intelligence, 17(6):635-640, June 1995.

[8] L. Robert and R. Deriche. Dense depth map reconstruction: A minimization and regularization approach which preserves discontinuities. In Proceedings of the Fourth European Conference on Computer Vision 1996, volume I, pages 439-451, Cambridge, April 1996.

[9] B. Serra and M. Berthod. Optimal subpixel matching of contour chains and segments. In IEEE Proceedings of the International Conference on Computer Vision, pages 402-407, Cambridge, Massachusetts, June 1995.

[10] J.A. Sethian. Level Set Methods: Evolving Interfaces in Geometry, Fluid Mechanics, Computer Vision and Materials Sciences. Cambridge University Press, 1996.

[11] T.Pajdla and L.Van Gool. Matching of 3d curves using semi differential invariants. In IEEE Proceedings of the International Conference on Computer Vision, pages 390-395, Cambridge, Massachusetts, June 1995. 